\title{
Possibility of Using the Spiral Single Mode Optical Fiber for Dispersion Compensation
}

\author{
Mohammed Yousef AL-Gawagzeh. \\ Department of Electrical power Engineering, Faculty of Engineering \\ Al-Balqa Applied University, JORDAN
}

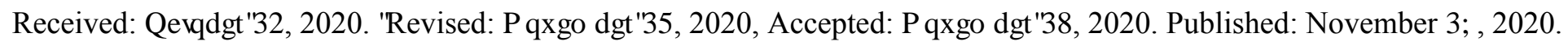

\begin{abstract}
-this research show the possibility of using the Anisotropy of spiral single mode optical fiber for compensation the main three types (material,waveguide,and polarized) of dispersion.it is also study the effect of energies mutual transformation of optical pulsed signals that carried by orthogonal waves $\left(\mathrm{HE}_{11}^{\mathrm{e}}\right)$ and $\left(\mathrm{HE}_{11}^{\mathrm{o}}\right)$ on the generation the chromatic and polarized dispersions and inter symbol distortions of signals.an example of polarized modal dispersion compensation is gives.
\end{abstract}

Keywords - Dispersion, Anisotropy, optical fiber, compensation.

\section{INTRODUCTION}

A TTENUATION and dispersion are the main problems of optical signals propagation through optical fiber transmission lines, the transmission speed and distance using optical amplifier are limited by dispersion such (material, waveguide,polarized),as well as other types of dispersion related to inhomogeneities of optical fiber path, under such conditions the problem of compensating the these types of dispersion in the amplifying and regenerating sections become more and more urgent.

Till now a significant number of methods and devices for dispersion compensation have been developed which directly induced in the linear path. The methods and devices of compensating the chromatic dispersion (material and waveguide) are reviewed in detail in $[1,4,8,9,10]$ and could be divide for two basics categories: -

1)-electrical methods which based on using more resistant to influence a variance of modulation formats, and linear filters with branched delay line.

2)- optical methods implemented based on various compensator circuits, which are included directly in the linear path of optical fiber transmission systems.

Both electrical and optical methods have advantages and disadvantages. The disadvantages of electrical method include the dependence of compensation degree on the transmission rate and information formats, less value of compensation comparing with optical method, dispersion limitation by on channel. But the described methods and devices are often quite complex, difficult to manufacture, and therefore rather expensive and sometimes difficult to interface with single mode optical fiber (SMOF). [7,9,13,14,15,16,17].

The purpose of this paper is to research the possibility of using the Anisotropy of spiral single mode optical fiber(SSMOF) to compensate the main three types of dispersion (material,waveguide, and polarized) taking in consideration the microstructure of quartz which form the basic rectilinear isotropic of ideal circular single mode optical fibers that consist from anisotropic molecules and other structural elements, in particular microcrystals, which their axes are randomly oriented in space, and the macroscopic anisotropy is smoothed out only statistically and the profile of dielectric constant $\varepsilon(\mathrm{r})$ in such single mode optical fiber with a perfect production technology can be considered symmetric around the axis and depends only on the radial coordinate $(r)$ and frequency $(\omega)$.

The main modes that carry information signals in such (SMOF) are two mutually orthogonal waves $\left(H E_{11}^{e}\right)$ and $\left(H E_{11}^{o}\right)$ with arbitrarily oriented of polarization in the cross section.

If the waveguide structure of (SMOF) is ideally symmetric, then both waves with mutually orthogonal polarization have the same propagation coefficient $\left(\beta_{01}\right)$,and they will transfer equal values of signal energy, such fibers are called (SMOF).however real optical fiber, and due to random industrial defects(ellipticity and eccentricity) of (SMOF) core section the axial asymmetry occurs when the propagation coefficients of two mutually orthogonal waves become different and in addition, in case of accidental bending of (SMOF) in practice during their laying in cable and disturbances due to temperature fluctuations and vibrations of the environment (when laying the optical cables along transport roads or railways) the anisotropy arises randomly and distributed along the length of (SMOF) and as a result the propagation velocities of these waves along the fiber length are differ, for this reason, the energies mutual transformation of optical pulsed signals that carried by these waves are changes along the fiber(the polarization of waves)and additionally generates the chromatic and polarized dispersions and inter symbol distortions of signals.

In work [2] it has been experimentally proved that the harmful effect of these random factors is partially eliminated due to manifestation of the photo elastic effect which regulates the molecular and microcrystal structure of the fiber in (SMOF) 
with its reasonable regular bending in spiral when stacked in optical cable. Thus, the problem of minimizing the random anisotropy in (SMOF) caused by random manifestations of chromatic dispersion of optical pulsed signals can be partially solved by creation an artificial anisotropy that related to mechanical stresses in (SMOF) and subsequent change in the dielectric constant tensor which arising when optical fiber laid in spiral form.

\section{Methods And Procedure}

In the cross sections of regularly spiral curved (SMOF) a stable distinguished directions of minimax values of deformations or dielectric permittivity will appear and determine the propagation polarization of mutually orthogonal waves[3] as a function $(A=p / 4 \pi R)$, where $(p)$ and $(R)$ are the step and radius of spiral curved axis of (SMOF) of the wave $\left(H E_{11}\right)$, the electric field in the cross section of (SMOF) that oriented along the selected directions of the minimax values of material deformations(compression or tension) due to spiral bent form will be called extraordinary and indexed as $\left(H E_{11}^{e}\right)$ or just $(\vec{e})$.and the wave $\left(H E_{11}^{o}\right)$ will be called ordinary or just $(\vec{o})$.then the polarization angles of ordinary( $\vec{o}$ )and extraordinary $(\vec{e})$.waves can be determined as:

$\varphi^{e}=-\operatorname{arctg}\left(\frac{p}{4 \pi R}\right)=-\operatorname{arctg}(A) ; \varphi^{o}=\varphi^{e} \pm \pi / 2$

The phase propagation coefficients $[4,11]$ defined as:

$\beta_{1}^{e}(r, \varphi)=\beta_{10} \sqrt{\frac{\varepsilon^{e}(r, \varphi)}{\varepsilon(r)}} ; \beta_{1}^{o}(r, \varphi)=\beta_{10} \sqrt{\frac{\varepsilon^{o}(r, \varphi)}{\varepsilon(r)}}$

Where $\left(\beta_{10}\right)$-the frequency dependent coefficient of $\left(H E_{11}\right)$ wave propagation before bending in (SMOF). $\left(\varepsilon^{e}(r, \varphi), \varepsilon^{e}(r, \varphi)\right)$ - the functions of dielectric permittivity for fundamental extraordinary and ordinary waves respectively in the planes of their polarizations, and determined by the dimensionless parameter $(A=p / 4 \pi R)$ which obtained in $[3,12]$

$\varepsilon(r)$ - frequency dependent of dielectric constant of (SMOF)before bending.

The phase coefficients for ordinary and extraordinary waves indicates the chromatic dispersions of the impulse signals and can be determined as the difference between wave group delay (propagation time) in accordance with [5] and written in polar coordinates in the form:

$$
\begin{aligned}
& \Delta \tau_{1}=\tau_{1}^{e}-\tau_{1}^{o}=\frac{\partial \beta_{1}^{e}}{\partial \omega}-\frac{\partial \beta_{1}^{o}}{\partial \omega} \approx \frac{1}{2} \frac{\partial}{\partial \omega}\left(\frac{\beta_{10}}{\varepsilon(r)}\right)[(v r- \\
& \left.2 \chi r) \cos \varphi+v r \sin \varphi+\chi^{2} r^{2} \cos \varphi\right]
\end{aligned}
$$

$$
\text { Where } \begin{aligned}
\cos (\operatorname{arctg} A) & =1 / \sqrt{1+A^{2}}
\end{aligned}
$$

$\left(\chi=\frac{1}{R\left(1+4 A^{2}\right)}\right) ; \quad\left(v=\frac{2 A}{R\left(1+4 A^{2}\right)}\right)-$ curvature and torsion respectively of the (SMOF) axis.

For partially coherent laser radiation source with frequency band $\left(\Delta \omega=2 \pi c \frac{\Delta \lambda}{\lambda^{2}}\right)$, where $(\Delta \lambda)$-the spectral line width, $(\lambda)$ central radiation wave length .

The expression for the polarized dispersion due to the helicity bending of (SMOF) can be written as:

$\Delta \sigma=\Delta \omega \frac{\partial\left(\Delta \tau_{1}\right)}{\partial \omega}=\frac{1}{2} \Delta \omega \frac{\partial^{2}}{\partial \omega^{2}}\left(\frac{\beta_{10}}{\varepsilon(r)}\right)[(v r-2 \chi r) \cos \varphi+$ $\left.v r \sin \varphi+\chi^{2} r^{2} \cos \varphi\right]$

$\varepsilon(r)=\sqrt{n(r)}$-spectral dependance of dielectric permittivity with wavelength range $(\lambda=0.2-2.0$ micron $)$, then its possible to describe the Selmeyer dispersion formula:

$\varepsilon(r)=n^{2}(r)=1+\sum_{i=1}^{3} a_{i} \lambda /\left(\lambda^{2}-l_{i}^{2}\right)$

Where $a_{i}, l_{i}$ - Selmeyer coefficients that depends on the chemical composition of fiber.

Taking in consideration $\frac{\partial}{\partial \omega}\left(\frac{\beta_{10}}{\varepsilon(r)}\right)$ and substituting into the expression (3), we can get a full expression for calculating the linear value of polarized dispersion that could be compensated in the coil:

$\Delta \tau_{c o i l}=F \frac{\sqrt{1+\sum_{i=1}^{3} \frac{a_{i} \cdot \lambda^{2}}{\lambda^{2}-l_{i}^{2}}}-\frac{\lambda^{2}}{n 1} \sum_{i=1}^{3} \frac{a_{i} \cdot l_{i}^{2}}{\left(\lambda^{2}-l_{i}^{2}\right)^{2}}}{2 . c\left(1+\sum_{i=1}^{3} \frac{a_{i} \cdot \lambda^{2}}{\left(\lambda^{2}-l_{i}^{2}\right)}\right)}$

Where $F-\left[(v r-2 \chi r) \cos \varphi+v r \sin \varphi+\chi^{2} r^{2} \cos \varphi\right]$

c- speed of light in vacuum.

$n_{1}$ - (SMOF) core step index.

Expression (6) give the possibility to determine the polarized modal dispersion which bent on spiral (SMOF) for different chemical composition. 
In table 1.shown an example results of calculations the polarized modal dispersion that may be compensated for wavelength $\lambda=1.55$ micron, where the material of (SMOF) core is $100 \%$ from $\mathrm{SiO}_{2}$. and the minimum radius of the core was taken $(5 \mathrm{~mm})$ which is the minimum permissible bending radius of the fiber with fiber laying of (250 micron) [8]. where the value of compensation will be depend basically on the length of fiber and core radius $\left(R_{\text {core }}\right)$.

Table.1: results of polarized modal dispersion

\begin{tabular}{|l|l|}
\hline$\left(R_{\text {core }}\right), \mathrm{mm}$ & $\Delta \tau_{\text {coil }}, \mathrm{ps} / \mathrm{km}$ \\
\hline 5 & -1639.83 \\
\hline 6 & -1367.48 \\
\hline 7 & -1172.71 \\
\hline 8 & -1026.49 \\
\hline 9 & -912.69 \\
\hline 10 & -821.61 \\
\hline
\end{tabular}

The total length of specific dispersion of pulsed signals in (SMOF) that laid in optical cable or dispersion compensator can be represented as the sum of chromatic dispersion $\left(\sigma_{c h r}\right)$ in rectilinear isotropic(SMOF) before bending it into a spiral form[4]

$\sigma_{c h r .}=\Delta \omega \frac{\partial^{2}}{\partial \omega^{2}}\left(\frac{\beta_{10}}{\varepsilon(r)}\right)$

The value $(\Delta \sigma)$ in accordance with (4) and due to bending and twisting when laying the cable in spiral form:

$$
\begin{aligned}
& \sigma=\sigma_{c h r .}+\Delta \sigma=\Delta \omega \frac{\partial^{2}}{\partial \omega^{2}}\left(\frac{\beta_{10}}{\varepsilon(r)}\right)\left\{1+\frac{1}{2}[(v r-2 \chi r) \cos \varphi+\right. \\
& \left.\left.v r \sin \varphi+\chi^{2} r^{2} \cos \varphi\right]\right\}
\end{aligned}
$$

\section{Result AND Discussion}

It is important to note that the difference of group delay (3)and change in the chromatic and polarization dispersion(4) taken together and due to the spiral bending and depending on the curvature and torsion of (SMOF), then a positive or negative or zero values can be acquire which determined by the expression $\left[(v r-2 \chi r) \cos \varphi+v r \sin \varphi+\chi^{2} r^{2} \cos \varphi\right]$ that can be used to correct (compensate)the dispersion of pulsed signals transmitted through (SMOF) containing significant irregularities and distort the phase frequency and amplitude frequency characteristics. These changes in the characteristics of (SMOF) are appear due to the spiral bent optical fiber and determined simultaneously by changes in both chromatic and polarized dispersions.

In addition, the above considered spatial frequency temporal changes in the signal must also be supplemented with changes that caused by transient processes between mutually orthogonal polarizations of waves that consisting in nonreciprocal exchange of energies for pulsed signals depending on the parameter $(A=p / 4 \pi R)[6,7]$.

In work [6], the nonreciprocal waves influence on (SMOF) are considered and the possibility of controlling the durations of ordinary and extraordinary waves depending on the parameter $(A)$ when laying the (SMOF) into an optical cable or optical dispersion compensator is shown. For example, when $(0<A<$ 0.3 ), then both the leading and trialing edges of the total signal carried simultaneously by both mutually orthogonal waves are shortened and carried out by a simultaneously decrease in both chromatic and polarized dispersions. And if it's necessary to compensate for example only polarized dispersion in the transmission path, then in this case a corrector- optical delay line (ODL) based on (SMOF) with $A \rightarrow 0$ is required [6] where both waves $\left(H E_{11}^{e}\right)$ and $\left(H E_{11}^{o}\right)$ will propagate differently and the transient influences between these waves will be absent and the difference between their group delays of pulse signals will be provided with a maximum in absolute value.

\section{CONCLUSION}

The bending of (SMOF) into spiral form which characterized by parameter $(0 \leq A=p / 4 \pi R<\infty)$ changes the parameters of dispersion distortions and based on the difference in signals and their changes, it is possible to synthesize a fiber optic path for transmitting pulsed signals with a minimum value of chromatic or polarized dispersion or with minimum value of intersymbol distortion.

Such solutions can be implemented in the form of an optical cable with the necessary values of the parameter $(A=p / 4 \pi R)$ of spiral (SMOF), or in the form of separate coil compensator of chromatic dispersion or in the form of a coil optical delay line of one of the mutually orthogonal waves that carrying the pulsed signal.

Finally, we can say that the potential for compensation of chromatic and polarized dispersion of impulse signals based on (SMOF) was discussed. 
Future work: based on the above results and conclusions, it is useful to study and research in future the possibility of using such compensator in the linear path of highspeed optical fiber multi-channel system of transmission with spectral multiplexing in the optical amplifiers, measurements schemes, etc.

\section{REFERENCES}

[1] M. Y. Al-Gawagzeh,."the effect of Anisotropy implementation on the components of optical transmission lines," IEEE Int. Conf. on information and Communication, 2004, pp.175-176.

[2] T. V. "Makarov, nonreciprocal effect waves and signals in single mode bended on spiral optical fiber."UNEERT, vol 1, pp.2329,2009.

[3] T. V. Makarov, "The transfer characteristic of bending optical fiber."UNEERT, vol 2, pp.29-34, 2010.

[4] T. V. "Makarov, Anisotropy of bending optical fiber," UNEERT vol 2, pp.103-108, 2006.

[5] N. P Zhuck, "Effective permittivity of a statistically inhomogeneous medium with strong permittivity fluctuations," Progress in Electromagnetic Research, PIER, vol 44, pp.169195, 2004.

[6] M. Y. AL-Gawagzeh, "Research the Dielectric permittivity of optical fiber."J.of electronic and electrical engineering, vol 1(89), pp.45 -49,2009.

[7] M. Y. Al-Gawagzeh, M. R. AL-Hadidi, R Rzooq,"Polarized modes Dispersion in Anisotropic Optical Fiber Communication lines," J.of Wseas Transaction on Communication, vol 8, pp.1120. 2009.

[8] G. Josselin."High-frequency asymptotic for Maxwell's equations in anisotropic media Part Nonlinear propagation and frequency conversion," journal of mathematical physics, vol 42, no 4, April 2001.

[9] M. Y AL-Gawagzeh, M. R AL-Hadidi, "Chromatic dispersion in fiber optic with ordered rotating of glass microstructure (ORGM),"J. of Optoelectronics and advanced materials-Rapid Communications, vol 8, pp.67-71. 2014.

[10] V. Kornechok, Optical Systems of Transfer, $3^{\text {rd }}$ ed., Radio and Communication Co.: Kiev. 2002.

[11] J.M Arnold, "Anisotropic Effect in non-linear optical waveguides," J .of wave motion, Vol 34, No.3, pp.339-352. 2001.

[12] A Snaider,"Theory of optical waveguides," (second Edition), Moscow, 223 pp. 2001.

[13] R. Kaur, M. Singh "A Review Paper on Dispersion Compensation Methods "IRJET, vol 4, no 6, pp.1991 -1994, June 2017.

[14] O. V Iorgach, "Losses on the Non-uniform Optical Fiber Cables," Journal of Foto- Electron, vol 11: pp. 61-64. 2011.

[15] M. Y AL-Gawagzeh,"Studying the Polarization Dispersion in the Bended on Spiral Optical Waveguide. ,"Journal of Engineering and Applied Sciences, Volume 13, issue 18: pp. 4971-4975. , 2018.

[16] P. Shukla, S. Tiwari, " A Review of Dispersion Compensation using Fiber Bragg Grating (FBG) in Optical Communication," International Advanced Research Journal in Science, Engineering and Technology, vol. 4, no. 6, pp. 66-69, June, 2017.
[17] N. Dalal , A. K. Garg," A Comprehensive Study of Various Compensation Techniques in High Speed Single Mode Optical Fiber Communication," International Journal of Recent Trends In Engineering and Research, vol. 02, no. 05, pp. 275-278, 2016.

\section{Creative Commons Attribution License 4.0 (Attribution 4.0 International, CC BY 4.0)}

This article is published under the terms of the Creative Commons Attribution License 4.0

https://creativecommons.org/licenses/by/4.0/deed.en_US 